\title{
Nutritional Quality of Breakfast Affects Cognitive Function: An fMRI Study
}

\author{
Yuko Akitsuki ${ }^{1,2 *}$, Seishu Nakawaga ${ }^{2 *}$, Motoaki Sugiura ${ }^{2}$, Ryuta Kawashima ${ }^{1,2}$ \\ ${ }^{1}$ Smart Ageing International Research Center, Institute of Development Aging and Cancer (IDAC), Sendai, Japan; ${ }^{2}$ Department of \\ Functional Brain Imaging, Institute of Development Aging and Cancer (IDAC), Sendai, Japan; \\ Email: fbi@idac.tohoku.ac.jp
}

Received May 19 ${ }^{\text {th }}, 2011$; revised June 20 $0^{\text {th }}, 2011$; accepted July $15^{\text {th }}, 2011$.

\begin{abstract}
To investigate the neural underpinnings of the effect of nutrition, brain activity of six young healthy volunteers who had a breakfast including various nutrients was compared to when they skipped breakfast or had only sugar for breakfast by functional magnetic resonance imaging. A repeated measure counterbalanced crossover design was employed. We demonstrated that significantly higher brain activation was observed in the medial aspect of the prefrontal cortex when the subjects had a nutritionally balanced breakfast while the subjects were conducting $N$-back tasks. This preliminary report was the first to demonstrate by means of brain imaging techniques that taking various nutrients as breakfast as well sugar has relevant impacts on underlying physiological events or cognition.
\end{abstract}

Keywords: Breakfast, Nutrition, Functional MRI, N-back Task, Prefrontal Activity

\section{Introduction}

Previous behavioral studies have shown the various aversive effects of skipping breakfast such as fatigue, a low body temperature, and decreased work efficiency [1]. Other evidence also suggests that breakfast consumption may improve cognitive function related to memory, test grades, and school attendance [2]. Furthermore, the presence of sugar is beneficial and a lack of dietary carbohydrates can indeed negatively influence cognitive performance and memory [3]. Based on these studies, Higuchi and colleagues conducted a behavioral study and demonstrated that a nutritionally well-balanced breakfast could contribute to the elevation of cognitive task efficiency [4].

To our knowledge, only one previous neuroimaging study investigated the relationship between brain activity measured by fMRI and ingestion of foods. Liu et al [5] demonstrated that oral ingestion of D-dextrose induced activation of the supplementary motor area, cerebellum, anterior cingulate and orbitofrontal cortex at the early phase, and deactivation of the medial hypothalamus at several minutes after the ingestion. They concluded that the formerly activated areas may be involved in the integration of sensory and visceral signals, and affective activity may be associated with appetite and taste or olfac-

*Both authors contributed equally. tion, however, the exact neurophysiological mechanisms underlying the negative response are unknown. Nevertheless, the neural basis of how the nutritional qualities of breakfast affect cognitive function is still unknown. In this experiment, we used fMRI techniques to investigate the relationship between cognitive function and the nutritional quality of breakfast. Water, sugar water, and a nutritionally balanced liquid food (NBLF) were used as breakfast.

\section{Methods}

\subsection{Subjects}

We recruited six healthy right-handed volunteers (Five males, mean age $\pm \mathrm{SD}=20.9$ years \pm 0.69 ), who were confirmed to be in the habit of eating nutritionally balanced breakfast every day. The protocol of this study was reviewed and approved by an institutional review board. Written informed consent was obtained from each subject.

\subsection{Task Procedures}

A repeated measure counterbalanced crossover design was employed for this study (Figure 1); each subject participated in this study three times, with a week break in between participation days. The subjects were instructed to have the same supper prepared for them (a 


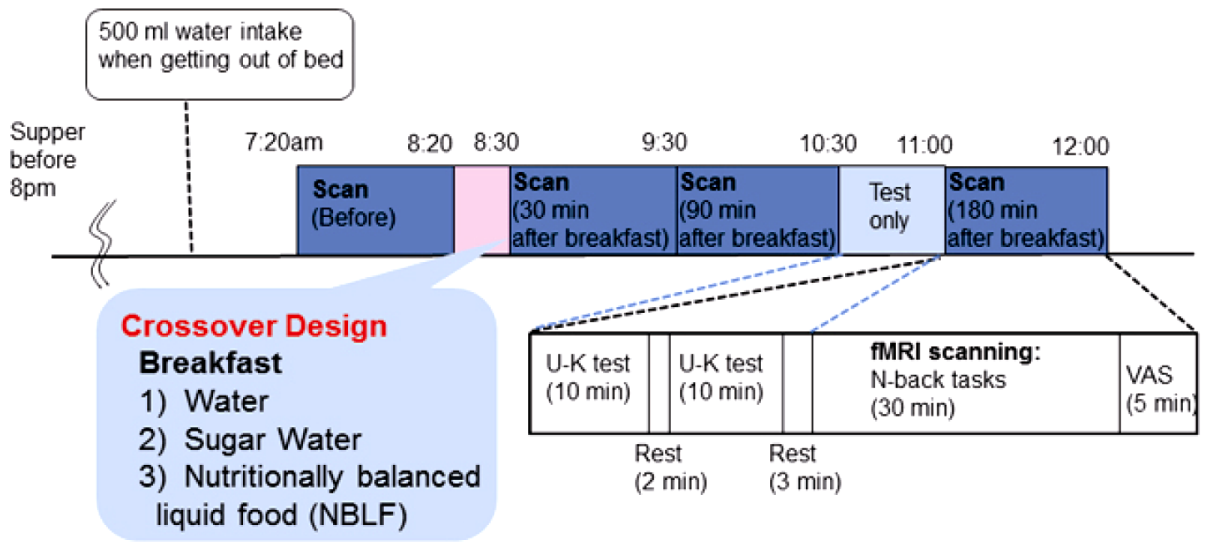

Figure 1. Experimental paradigm. A crossover design was used. Each subject participated in this study three times and had one of the following three types of breakfast: water, sugar water, and NBLF. On the experiment day, the subjects were instructed to perform a modified version of the Uchida-Kraepelin test (U-K test: a serial addition test) and N-back tasks (0back, 2back, 3back and 4 back). The subjects were scanned while they were performing the $\mathrm{N}$-back tasks before breakfast and at the following time points: $30 \mathrm{~min}, \mathbf{9 0} \mathrm{min}$, and $180 \mathrm{~min}$ after the consumption of breakfast. At each time point, the subjects were instructed to indicate their level of hunger, fatigue, and concentration by using a visual analogue scale (VAS).

packed box lunch with steamed rice, meat, fish, and vegetables; $3698.7 \mathrm{~kJ} /$ supper) before $8 \mathrm{pm}$ on the evening of the day before the experiment. After an overnight fast, on the experiment day, the subjects were instructed to take $500 \mathrm{ml}$ water when they got up in order to avoid dehydration. Then, the subjects came to the lab and were instructed to perform a modified version of the UchidaKraepelin test (U-K test: a serial addition test, one set consists of two 10 min-tests with 2 min rests in-between). The U-K test was employed to induce mental fatigue and to evaluate the level of cognitive performance [6,7].

Following that, the subjects were scanned with a 3.0 Tesla MRI scanner (Philips Achieva Quasar Dual, Philips Medical Systems, Best, The Netherlands) while they were conducting N-back tasks (0back, 2back, 3back and 4back) $[8,9]$. During fMRI, stimuli were displayed on a small screen attached to the head coil of the scanner. The test stimuli were numbers (i.e. 1 to 4 ) that were presented one at a time. Each stimulus appeared for $0.5 \mathrm{~s}$ and the inter stimulus interval was $0.7 \mathrm{~s}$. The participants were presented with a series of stimuli and were asked to memorize them and their temporal order. That is, the subjects were continuously required to push one of four buttons that corresponded to the stimuli that were shown $\mathrm{N}$ stimuli ago during the task period. A standard block design was used; each session consisted of twelve 21.6sec blocks. There was a 1.9-sec instruction screen before each task block and a 14-sec block of rest after each task block. During rest, subjects were instructed to fix their eyes on a cross that was displayed at the center of the screen. Each subject completed two 8-min sessions per run. The sequence of the presentation of the N-back block types was counterbalanced among subjects.
Then, the subjects had one of the following three types of breakfast: 1) $500 \mathrm{ml}$ water, 2) $500 \mathrm{ml}$ sugar water containing 100 grams dextrin (1673.6 kJ), and 3) NBLF: a commercial food product ( $($ Calorie Mate, Otsuka Pharmaceutical Co., Ltd., Tokyo, Japan: a liquid type nutritional supplement; $400 \mathrm{ml} ; 1673.6 \mathrm{~kJ}$ ) with $100 \mathrm{ml}$ water. This food product contains, not only carbohydrates (sugar, dextrin, and lactose; total $67 \mathrm{~g}$ ), but also proteins (15.2 g), lipids (8.8 g), vitamins (Vitamin A 450 $\mu \mathrm{g}$, Vitamin B1 $1 \mathrm{mg}$, Vitamin B2 $1.2 \mathrm{mg}$, Vitamin B6 1 $\mathrm{mg}$, Vitamin B12 $2 \mu \mathrm{g}$, Niacin $11 \mathrm{mg}$, Pantothenic acid $5.6 \mathrm{mg}$, Folic acid $200 \mu \mathrm{g}$, Vitamin C $80 \mathrm{mg}$, Vitamin D $5 \mu \mathrm{g}$ and Vitamin E $8 \mathrm{mg}$ ) and minerals (Sodium $680 \mathrm{mg}$, Potassium $340 \mathrm{mg}$, Calcium $140 \mathrm{mg}$, Iron $2.6 \mathrm{mg}$, Magnesium $50 \mathrm{mg}$ and Phosphorus $240 \mathrm{mg}$ ). The food's energy ratio of protein, fat and carbohydrates is 15:20:65. The order of the breakfast type was counterbalanced among the subjects. After breakfast, the subjects were instructed to repeatedly perform the U-K test and the Nback tasks. The subjects were scanned while they were performing the N-back tasks at the following time points: $30 \mathrm{~min}, 90 \mathrm{~min}$, and $180 \mathrm{~min}$ after the consumption of breakfast. Before and after breakfast (at the above mentioned time points), the subjects were instructed to indicate their levels of hunger, fatigue, and concentration by using a visual analogue scale (VAS).

\subsection{Brain Imaging}

Functional images were obtained using a $\mathrm{T} 2{ }^{*}$-weighted gradient-echo echo-planer imaging sequence (TE/TR: 30/ 2500 ms, matrix: $64 \times 64$, FOV $192 \mathrm{~mm}$, Forty-two 3 mm-thick axial slices).

Imaging data were preprocessed and analyzed using 
SPM5 (Welcome Department of Cognitive Neurology, London, UK) in MATLAB (Mathworks, Sherborn, MA). While our experimental design was three factorial (i.e., breakfast type $\times$ time $\times$ n-back), the number of our subject was too small for a three-way ANOVA approach (i.e., insufficient number of images to calculate covariance components). Therefore, to search for the cortical regions affected by breakfast type, we focused to the 4back condition in which the effect of breakfast type was expected to be largest.

A standard two-stage random effect approach was employed. First, image representing change from "before" (prior to breakfast) to 4 back task at $30 \mathrm{~min}, 90 \mathrm{~min}$, or 180 min compared with that at was calculated for each subject for each breakfast type. Then, a two-way repeated measure analysis of variance (AVONA; breakfast type (water, sugar, NBLF) $\times$ time $(30$ min-before, 60 min-before, 180 min-before)) was conducted as secondlevel analysis. Since we were interested in the main effect of breakfast type and interaction, corresponding $\operatorname{SPM}\{\mathrm{F}\}$ s were thresholded at $p<0.001$ in height and 100 voxels in cluster size.

Because we found a region exhibiting a significant main effect of breakfast type (see Results), the following contrasts were post hoc tested (i.e., t-test): NBLF vs. (sugar + water), and (NBLF + sugar) vs. water. These contrasts were intended for higher activity specifically for NBLF, and those common for NBLF and sugar, respectively. Results were thresholded at $p<0.001$ in height, then for multiple comparisons corrected to $p<$ 0.05 using cluster size.

The breakfast-type effect was also examined in each $\mathrm{N}$-back condition in each identified region of interest (ROI) at the local maxim of the result of those contrasts to see whether the same tendency would be observed. In addition, the mean activation during the 4-back task compared with the rest was calculated at the local maxima of each breakfast type.

\section{Results}

\subsection{Behavioral Data}

The behavioral data showed that there was no significant difference in the accuracy rate of the U-K test and the accuracy rate of the N-back tasks across breakfast types at any given time point. However, the results of the VAS showed significant differences (Figure 2). As for the subjective feeling of hunger, when the subjects had NBLF for breakfast they felt significantly less hunger (Wilcoxon test, $p=0.046$ ) at $30 \mathrm{~min}$ after breakfast as compared to the pre (prior to breakfast). On the other hand, when the subjects had water for breakfast they felt significantly more hunger $(p=0.028$ at $90 \mathrm{~min}$ after breakfast, $p=0.028$ at $180 \mathrm{~min}$ after breakfast). When the subjects had sugar water for breakfast they felt significantly more hunger ( $p=0.028$ at $180 \mathrm{~min}$ after breakfast). As for fatigue, there was no significant difference compared to the baseline when the subject had NBLF. However, when the subjects had water or sugar water for breakfast, more fatigue ( $p=0.046$ at $90 \mathrm{~min}$ after breakfast, $p=0.075$ at $180 \mathrm{~min}$ after breakfast $)$ or $(p=0.046$ at 90 min after breakfast, $p=0.075$ at 180 min after breakfast), respectively, as compared to the pre. As for concentration, there was no significant difference compared to the baseline when the subject had NBLF. However, when the subjects had water or sugar water for breakfast, less able to concentrate ( $p=0.075$, at 90 min after breakfast) or ( $p=0.075$, at $90 \mathrm{~min}$ after breakfast), respectively, as compared to the pre.

What is more, a group difference was also found. The results of the VAS at the time point 90 min after breakfast indicated that when the subjects had NBLF for

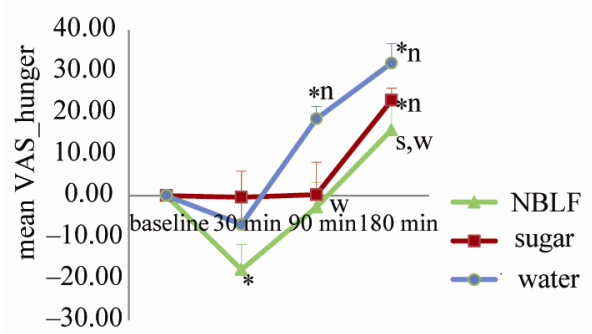

(a)

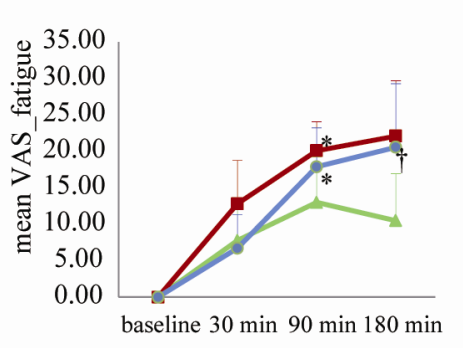

(b)

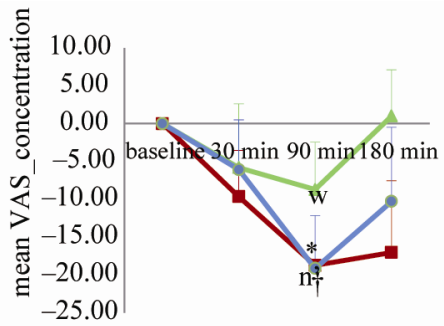

(c)

Figure 2. The results of VAS data. Each line indicates the mean change of the VAS score \pm SE as compared to the score at the baseline (i.e. delta from the baseline) at each time point. (a) Level of hunger: higher score indicates that the subjects felt stronger hunger, (b) Level of fatigue: higher score indicates that the subjects felt stronger fatigue, and (c) Level of concentration: higher score indicates that the subjects felt more able to concentrate. Wilcoxon tests were performed. A significant difference compared to the baseline was indicated as $* P<0.05$ and $\dagger P<0.01$. A significant group difference at each time point compared to the other breakfast types was indicated as n: NBLF $(P<0.05)$, s: Sugar water $(P<0.05)$, and w: Water $(P<$ 0.05). 
breakfast they felt significantly less hunger (Wilcoxon test, $p=0.046)$ and more able to concentrate $(p=0.059$, significant trend) as compared to when they had water for breakfast. In addition, the results of the VAS at the time point $180 \mathrm{~min}$ after breakfast showed that when the subjects had NBLF for breakfast they felt significantly less hunger as compared to when they had water $(p=$ $0.028)$ or sugar water $(p=0.046)$ for breakfast.

\section{2. fMRI Data}

Statistically significant main effect of breakfast type was found in the medial aspect of the prefrontal cortex (mPFC) (two foci; MNI coordinate; $\mathrm{x}, \mathrm{y}, \mathrm{z}=8,62,14$; $-12,58,22)$, and in the lateral surface of the superior frontal gyrus (SFG) (x, y, z =-18, 58, 22) (Figure 3(a)). The post hoc test revealed significant activation in this cluster specifically higher for NBLF (one located in the mPFC; $x, y, z=-2,54,6$, another located in the FSG; $x$, $\mathrm{y}, \mathrm{z}=-20,58,20)$ (Figure 3(b)), as well as those common for NBLF and sugar in the $\operatorname{mPFC}(\mathrm{x}, \mathrm{y}, \mathrm{z}=8,62,14)$ (Figure 3(c)). However, statistically significant interaction (breakfast type $\times$ time) was not identified. The ROI analysis demonstrated that the same tendency was observed at each time point (Figures 3(b) and (c)), that the significant activation of those areaa did not simply reflect the VAS scores since the time course of each VAS (Figure 2) and that of activation profile at the local maxima (Figures 3(b) and (c)) showed entirely the different patterns, and that those areas did not play a crucial role in performing the 4-back task since those areas were not activated (in most cases deactivated) during the 4-back task compared with the rest.

\section{Discussion}

To our knowledge, this is the first study investigate relationship between ingestion of foods and human brain
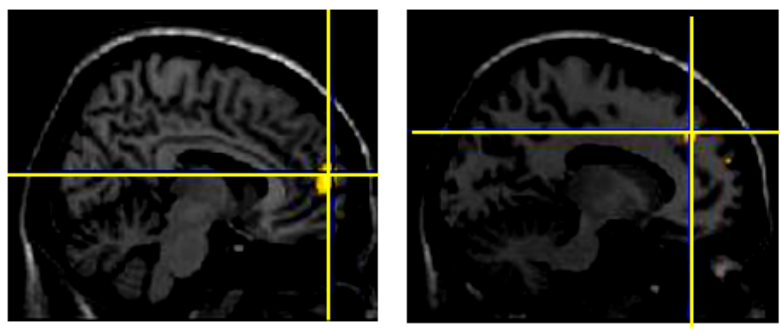

(a)
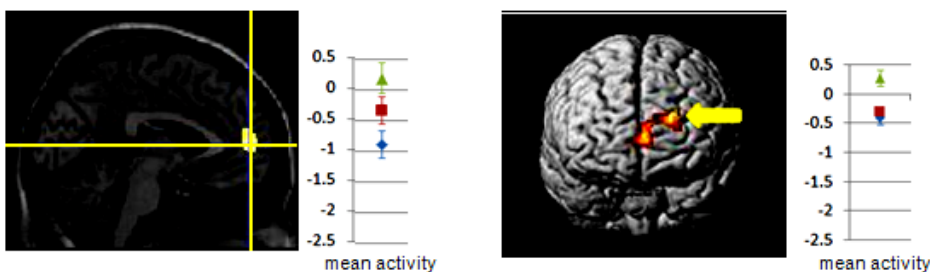

Lt. $\mathrm{mPFC}(-2546)$
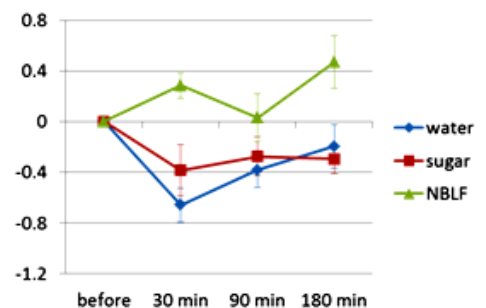

(b)

Lt. SFG $(-205820)$

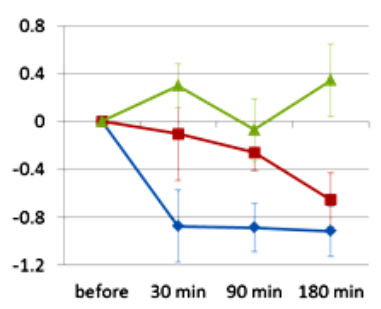

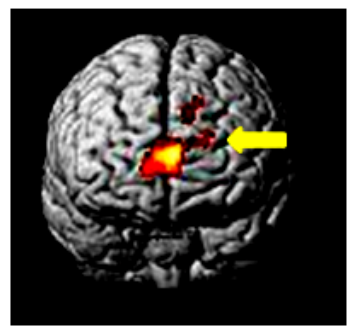
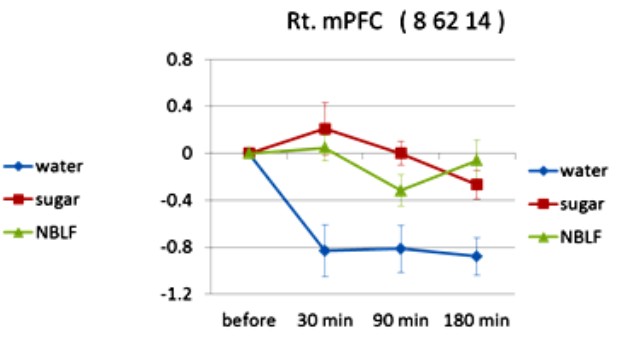

(c)

Figure 3. Main effect of breakfast. (a): A brain area in the medial aspect of the prefrontal cortex extending to the lateral surface of the superior frontal gyrus shows statistically significant main effect of breakfast type determined by SPM\{F\}s. (b), (c): Brain areas showing statistically significant activation in the contrast of NBLF vs. (sugar + water) (Figure 3(b)), and (NBLF + sugar) vs. water (c). Yellow cross lines and arrows indicate location of local maxima. Right graphs show the mean activation during the 4-back task compared with the rest at the local maxima of each breakfast type. Lower graphs show the activation profile at the local maxima at each time point. NBLF, mPFC, SFG, Lt. and Rt. indicate nutritionally balanced liquid food, medial aspect of the prefrontal cortex, superior frontal gyrus, left and right, respectively. 
activity. Glucose is the primary source of energy for the brain [10]. In our study, however, the subjective feelings of hunger, fatigue and concentration differed depending on the quality of breakfast. Moreover, significantly higher brain activation was observed in the mPFC extending to the SFG when the subjects had a nutritionally balanced breakfast, as compared to when they had only sugar for breakfast. Thus, blood glucose itself may not be the most relevant marker for the underlying physiological events or impacts of cognition [11]. In fact, a behavioral study has suggested that the overall postprandial cognitive performance of healthy young men in the morning is better after a balanced or protein-rich meal than it is after a high to medium glycemic carbohydrate-rich meal, and that pure carbohydrate was worse than other nutrients [12]. There is also a report demonstrating that better cognitive performance is observed after mixed meals compared to meals particularly rich in a single macronutrient [13]. Taken together, our data indicates the advantage of having a nutritionally well-balanced breakfast over skipping breakfast or only having something sugary for breakfast. This is consistent with the results of recent observational studies that report adequate intake of vitamins and minerals to be associated with better cognitive performance in a normally aging sample [14] and in children $[15,16]$. Therefore, our findings may well contribute to a public health recommendation for broad range of generations.

As for the fMRI data, brain activation in the mPFC extending to the SFG was associated with the quality of breakfast. Our results may be in line with the results of a previous imaging study reporting that dysfunction of the $\mathrm{mPFC}$ is associated with chronic fatigue [17]. Another interpretation for our results can be taken from task-induced deactivation (TID). TID refers to a regional decrease in blood flow during an active task relative to a "resting" or "passive" baseline [18]. Interestingly, previous behavioral and fMRI results indicate that the TID magnitude increases with task difficulty or increasing task processing demands in the default mode network (DMN) including the mPFC $[18,19]$. For example, as activity within the DMN increases, normalized activity in the external attention system shows decreased activity. These strong negative activities that correlate between the DMN and other systems is referred to as "dynamic equilibrium" or "anti-correlations" [20,21]. The nutritional quality of breakfast may affect these anti-correlations. That is, when the subject had a nutritionally balanced breakfast, their brain could work efficiently with less energy and thus the task demand was relatively small. This led to less deactivation in the mPFC in the DMN as compared to when the subjects had only water or only sugar water. In other words, with ill-balanced nutrition, their brains could not work efficiently and had to make more "efforts" (i.e. in terms of allocation of more blood volume from the DMN to the task related regions) to meet the same cognitive performance as they had with well-balanced nutrition. Therefore, the difference of the subjective feelings of hunger, fatigue, and concentration may have reflected the difference of the efficiency of the brain.

In sum, our data indicates that mere breakfast consumption is not sufficient but that nutritional quality of breakfast is relevant for better work efficiency. Further investigation will be needed for revealing the detailed mechanism of the association between multiple nutritional factors and various cognitive functions in order to provide better and precise public health recommendations in our society.

\section{Conclusions}

In this experiment, we used fMRI techniques to investigate the neural underpinnings of the effect of nutrition on young healthy volunteers who had a breakfast including various nutrients (i.e. food containing proteins, lipids, vitamins and minerals as well as carbohydrate) as compared to when they skipped breakfast or had only sugar for breakfast. We demonstrated that subjective feelings of hunger, fatigue and concentration differed depending on the quality of breakfast. Moreover, while the subjects were conducting N-back tasks, significantly higher brain activation was observed in the medial aspect of the prefrontal cortex when the subjects had a nutritionally balanced breakfast, as compared to when they skipped breakfast or had only sugar for breakfast. Sugar, especially glucose is the primary source of energy for the brain. We, however, were the first to demonstrate by means of brain imaging techniques that taking various nutrients as breakfast as well sugar has relevant impacts on underlying physiological events or cognition. Thus, it is of general interest for the public as a health recommendation that having a nutritional breakfast is necessary in order to work efficiently.

\section{Acknowledgements}

Funding for research presented in this paper was provided by Otsuka Pharmaceutical Co., Ltd. The roll of the funding source is to cover the cost for volunteer recruitment and MRI scanning. Otsuka Pharmaceutical Co., Ltd. also provided the food materials used in the experiment presented in this paper. The opinions expressed herein are those of the authors and do not necessarily reflect the views of Otsuka Pharmaceutical Co., Ltd. The funding organization did not influence the conduct or outcomes of the analysis or exercise any editorial control over this 
paper.

\section{REFERENCES}

[1] D. Benton and P. Y. Parker, "Breakfast, Blood Glucose, and Cognition," The American Journal of Clinical Nutrition, Vol. 67, No. 4, 1998, pp. S772-S778.

[2] G. C. Rampersau, M. A. Pereira, B. L. Girard, J. Adams and J. D. Metzl, "Breakfast Habits, Nutritional Status, Body Weight, and Academic Performance in Children and Adolescents," Journal of the American Dietetic Association, Vol. 105, No. 5, 2005, pp. 743-760.

doi:10.1016/j.jada.2005.02.007

[3] A. Hoyland, C. L. Lawton and L. Dye, "Acute Effects of Macronutrient Manipulations on Cognitive Test Performance in Healthy Young Adults: A Systematic Research Review," Neuroscience \& Biobehavioral Reviews, Vol. 32, No. 1, 2007, pp. 72-85. doi:10.1016/j.neubiorev.2007.05.006

[4] T. Higuchi, K. Hamada, S. Imazuya and S. Irie, "The Effect of Breakfast Omission and Breakfast Type on Body Temperature, Mood and Intellectual Performance," Journal of Japanese Society of Clinical Nutrition, Vol. 29, No. 1, 2007, pp. 35-43.

[5] Y. Liu, J. H. Gao, H. L. Liu and P. T. Fox, "The Temporal Response of the Brain after Eating Revealed by Functional MRI," Nature, Vol. 405, No. 6790, 2000, pp. 10581062. doi: $10.1038 / 35016590$

[6] M. Matsuda and F. Matsuda, "The Factorial Analysis of Uchida-Kraepelin Pshychodiagnostic Test in Normal and Delinquent Boys and Girls," Japanese Journal of Educational Psychology, Vol. 16, 1968, pp. 50-58.

[7] K. Sugimoto, A. Kanai and N. Shoji, "The Effectiveness of the Uchida-Kraepelin Test for Psychological Stress: An Analysis of Plasma and Salivary Stress Substances," BioPsychoSocial Medicine, Vol. 3, No. 5, 2009. Art ID: 5. http://www.bpsme-dicine.com/content

[8] W. K. Kirchner, "Age Differences in Short-Term Retention of Rapidly Changing Information," Journal of Experimental Psychology, Vol. 55, No. 4, 1958, pp. 352358. doi: $10.1037 / \mathrm{h} 0043688$

[9] M. J. Susanne and B. Martin, "The Concurrent Validity of the N-Back Task as a Working Memory Measure," Memory, Vol. 18, No. 4, 2010, pp. 394-412. doi:10.1080/09658211003702171

[10] R. J. Berger, and N. H. Philips, "Comparative Aspects of Energy Metabolism, Body Temperature and Sleep," Acta physiologica Scandinavica Supplement, Vol. 574, 1998, pp. 21-27.

[11] E. A. de Bruin and M. B. Gilsenan, "Effects of Food Energy on Cognitive Performance: No Support from EventRelated Potentials," British Journal of Nutrition, Vol. 101, No. 7, 2009, pp. 1047-1051. doi:10.1017/S0007114508051702

[12] K. Fischer, P. C. Colombani, W. Langhans and C. Wenk,
"Carbohydrate to Protein Ration in Food and Cognitive Performance in the Morning," Physiology \& Behavior, Vol. 75, No. 3, 2002, pp. 411-423. doi:10.1016/S0031-9384(01)00676-X

[13] A. Cunliffe, O. A. Obeid and T. J. Powell, "Post-Prandial Changes in Measures of Fatigue: Effect of a Mixed or a Pure Carbohydrate or Pure Fat Meal," European Journal of Clinical Nutrition, Vol. 51, No. 12, 1997, pp. 831-838. doi:10.1038/sj.ejen.1600496

[14] A. La Rue, K. M. Koehler, S. J. Wayne, S. J. Chiulli, K. Y. Haaland and P. J. Garry, "Nutritional Status and Cognitive Functioning in a Normally Aging Sample: a 6-y Reassessment," The American Journal of Clinical Nutrition, Vol. 65, No. 1, 1997, pp. 20-29.

[15] A. Eilander, T. Gera, H. S. Sachdev, C. Transler, H. C. van der Knaap, F. J. Kok and S. J. Osendarp, "Multiple Micronutrient Supplementation for Improving Cognitive Performance in Children: Systematic Review of Randomised Controlled Trials," The American Journal of Clinical Nutrition, Vol. 91, No. 1, 2010, pp. 115-130. doi:10.3945/ajen.2009.28376

[16] C. F. Haskell, A. B. Scholey, P. A. Jackson, J. M. Elliott, M. A. Defeyter, J. Greer, B. C. Robertson, T. Buchanan, B. Tiplady and D. O. Kennedy, "Cognitive and Mood Effects in Healthy Children during 12 Weeks' Supplementation with Multi-Vitamin/Minerals," British Journal of Nutrition, Vol. 100, No. 5, 2008, pp. 1086-1096. doi:10.1017/S0007114508959213

[17] S. Yamamoto, Y. Ouchi, H. Onoe, E. Yoshikawa, H. Tsukada, H. Takahashi, M. Iwase, K. Yamaguchi, H. Kuratsune and Y. Watanabe, "Reduction of Serotonin Transporters of Patients with Chronic Fatigue Syndrome," Neuroreport, Vol. 15, No. 17, 2004, pp. 25712574. doi:10.1097/00001756-200412030-00002

[18] K. A. Mckiernan, J. N. Kaufman, J. Kucera-Thompson and J. R. Binder, "A Parametric Manipulation of Factors Affecting Task-Induced Deactivation in Functional Neuroimaging," Journal of Cognitive Neuroscience, Vol. 15, No. 3, 2003, pp. 394-408. doi:10.1162/089892903321593117

[19] R. L. Buckner, J. R. Andrews-Hanna and D. L. Schacter, "The Brain's Default Network Anatomy, Function, and Relevance to Disease," Annals of the New York Academy of Sciences, Vol. 1124, 2008, pp. 1-38. doi:10.1196/annals.1440.011

[20] M. D. Greicius, B. Krasnow, A. L. Reiss and V. Menon, "Functional Connectivity in the Resting Brain: A Network Analysis of the Default Mode Hypothesis," Proceedings of the National Academy of Sciences of the USA, Vol. 100, No. 1, 2003, pp. 253-258. doi: $10.1073 /$ pnas.0135058100

[21] P. Fransson, "Spontaneous Low-Frequency BOLD Signal Fluctuations: An fMRI Investigation of the Resting-State Default Mode of Brain Function Hypothesis," Human Brain Mapping, Vol. 26, No. 1, 2006, pp. 15-29. doi: $10.1002 / \mathrm{hbm} .20113$ 